\title{
ArcheoSciences
}

Revue d'archéométrie

\section{Quand l'aluminium valait de l'or: peut-on reconnaître un aluminium « chimique » d'un aluminium « électrolytique »?}

David Bourgarit et Jean Plateau

\section{(2) OpenEdition}

Journals

Édition électronique

URL : http://journals.openedition.org/archeosciences/560

DOI : 10.4000/archeosciences.560

ISBN : 978-2-7535-1594-9

ISSN : $2104-3728$

Éditeur

Presses universitaires de Rennes

\section{Édition imprimée}

Date de publication : 31 décembre 2005

Pagination : 95-105

ISSN : 1960-1360

Référence électronique

David Bourgarit et Jean Plateau, «Quand l'aluminium valait de l'or : peut-on reconnaître un aluminium " chimique » d'un aluminium «électrolytique »? », ArcheoSciences [En ligne], 29 | 2005, mis en ligne le 31 décembre 2007, consulté le 11 février 2021. URL : http://journals.openedition.org/archeosciences/ $560 ;$ DOI : https://doi.org/10.4000/archeosciences.560 


\title{
Quand l'aluminium valait de l'or : peut-on reconnaître un alumi- nium « chimique » d'un aluminium « électrolytique »?
}

\author{
David BOURGARIT* et Jean PLATEAU**
}

\begin{abstract}
Résumé : A partir de 1854, Sainte-Claire Deville développe progressivement un procédé chimique industriel de fabrication de l'aluminium fondé sur la réduction du chlorure d'aluminium par le sodium. Le nouveau métal fait sensation et rivalise avec les métaux les plus précicux : bijoux, objets de prestige sont alors fabriqués, ct Napolćon III en personne passe commande. Il faut dire que pendant une trentaine d'années, l'aluminium reste cher à produire et donc rarc. La France est alors pratiquement seule à en fabriquer. Ce n'est qu'à partir de 1890 que le procédé électrolytique encore en vigueur aujourd'hui est mis au point. Il remplace le procédé chimique et autorise une production de masse : l'aluminium perd ses lettres de noblesse. Ce faisant, l'aluminium entre dans l'histoire des techniques, selon deux grandes périodes, l'âge « chimique » et l'âge « ćlectrolytique ».

L'attribution d'un objet en aluminium à l'unc ou l'autre période par la sculc typologic s'avère parfois peu satisfaisante. L'objectif du présent travail est de tenter de mettre au point un critère de datation fondé sur la composition élémentaire. A cet cffet, plusicurs campagnes d'analyse par PIXE ont été menées sur 68 médailles, bijoux et objets emblématiques bien datés, couvrant une période comprise entre 1855 et 1916. Les résultats ont dépassé nos attentes, puisque pas moins de 6 éléments chimiques sont apparus comme discriminants : le $\mathrm{Mn}, \mathrm{Fe}, \mathrm{Pb}$ et dans une moindre mesure le $\mathrm{Cr}, \mathrm{Zr}, \mathrm{Ag}$ affichent des teneurs majoritairement supéricures dans les aluminiums élaborés par le procédé chimique. Ces résultats sont discutés, et le nouvcau critèrc proposé est testé sur un corpus de 42 objets supplémentaires plus ou moins bien datés par leur typologic.
\end{abstract}

Abstract: From 1854 onward, Sainte-Claire Deville progrcssivcly develops an industrial chemical process for aluminium production, based on aluminium chloride reduction by sodium. The new metal causes a sensation and soon competes with most precious metals: jewellery and prestigious items are produced, some being ordered by Napolcon III himself. The cost of aluminium production indeed remains high during the first 30 years, thus making this metal rare. France is then the only producer. One must wait until 1890 for the modern clectrolytic process to be settled and to replace the chemical process, thus leading to low-cost mass production. Hence, aluminium enters the history of techniques along two main periods, the "chemical age" and the "clectrolytic age". Dating an aluminium object to one or the other "age" according to its sole typology may be in some cases hazardous. The present work aims at setting up a new criterion based on elemental composition. Therefore, some 68 surely dated medals, jewels, and emblematic items ranging from 1855 to 1916 have been analysed by PIXE. Six chemical elements thus happen to discriminate: the contents of $\mathrm{Mn}, \mathrm{Fe}, \mathrm{Pb}$ and to a lesser extent $\mathrm{Cr}, \mathrm{Zr}, \mathrm{Ag}$ are generally higher in the aluminium produced by the chemical process. These results are discussed and the new eriterion is tested on some $\mathbf{4 2}$ additional objects more or less well dated by their typology.

Mots-clés : Aluminium, PIXE, composition élémentaire, authentification.

Key-words: Aluminium, PIXE, elemental composition.

\section{Introduction}

Si l'aluminium est aujourd'hui universellement fabriqué par électrolyse, s'il en est consommé plus de 30 millions de tonnes par an dans le monde, il a d'abord été obtenu beaucoup plus modestement par un procédé chimique, fondé sur la réduction du chlorure d'aluminium par le sodium (Plateau, 2003). Ce procédé a été adapté à l'échelle industrielle par Henri Sainte-Claire Deville, entre 1854 et 1860 . Puis, de 1860 à 1890 , la Compagnie des Produits Chimiques d'Alais et de la Camargue, dans son usine de Salindres, fut à peu près la seule au monde à produire de l'aluminium, une quarantaine de tonnes au total.
Les premiers kilos d'aluminium produits coûtent aussi cher que l'or, puis son prix de revient diminue progressivement. On utilise alors le nouveau métal pour faire des objets prestigieux (fig. 1), tels que les bracelets que réalise Honoré Bourdoncle (1858, Musée des Arts Décoratifs) ou le groupe de Putti offert par Christofle à Napoléon III (1858, Musée de Compiègne). En 1860, l'empereur, soucieux de diminuer la charge des porte-drapeaux, commande à Salindres (Gard) 217 aigles en aluminium destinées au sommet des hampes des drapeaux. Le musée de l'Armée en conserve quelques exemplaires.

Mais, même à Salindres, où la proximité des matières premières est un facteur favorable, on ne parvient pas à

\footnotetext{
* Centre de Recherche et de Restauration des Musées de France, Palais du Lotnre - Porte des Lions, 14 quai François Mitterrand, 75001 PARIS, e-mail : david.bourgarit()culture.gouv.fr (auteur de correspondance).

** Membre du Conseil de l'Institut pour l'Histoire de l'Aluminium, 4 ne de la croix, Cidex 8463,41000 St DENIS/LOIRE, e-mail : jla.p@wanadoo.fr
} 

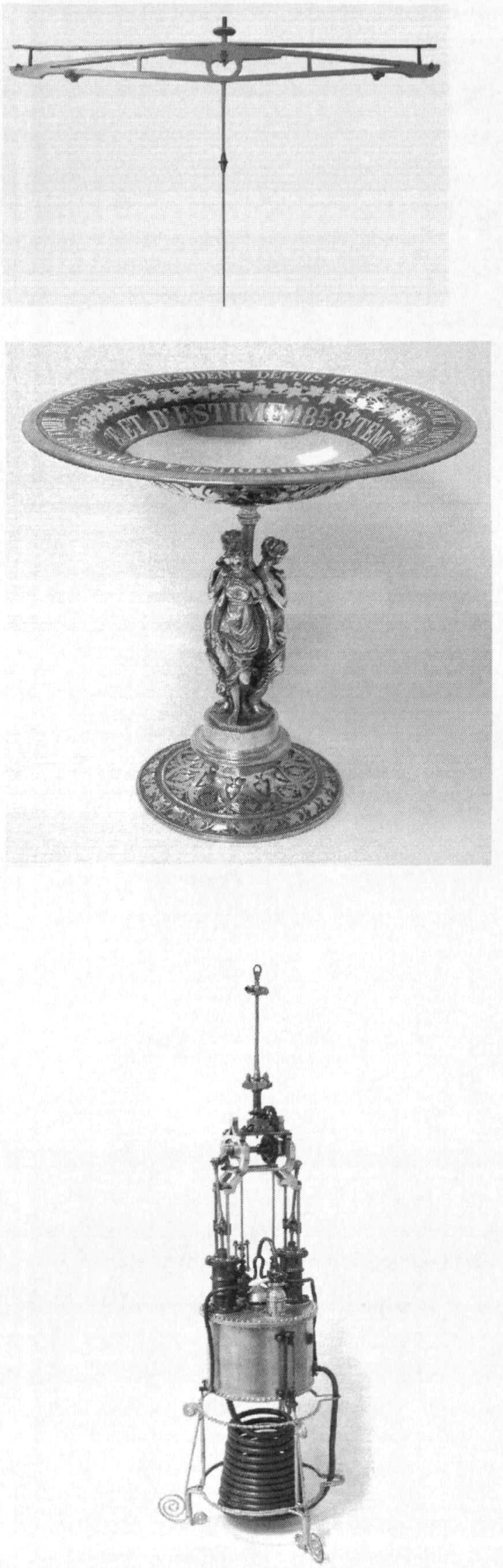
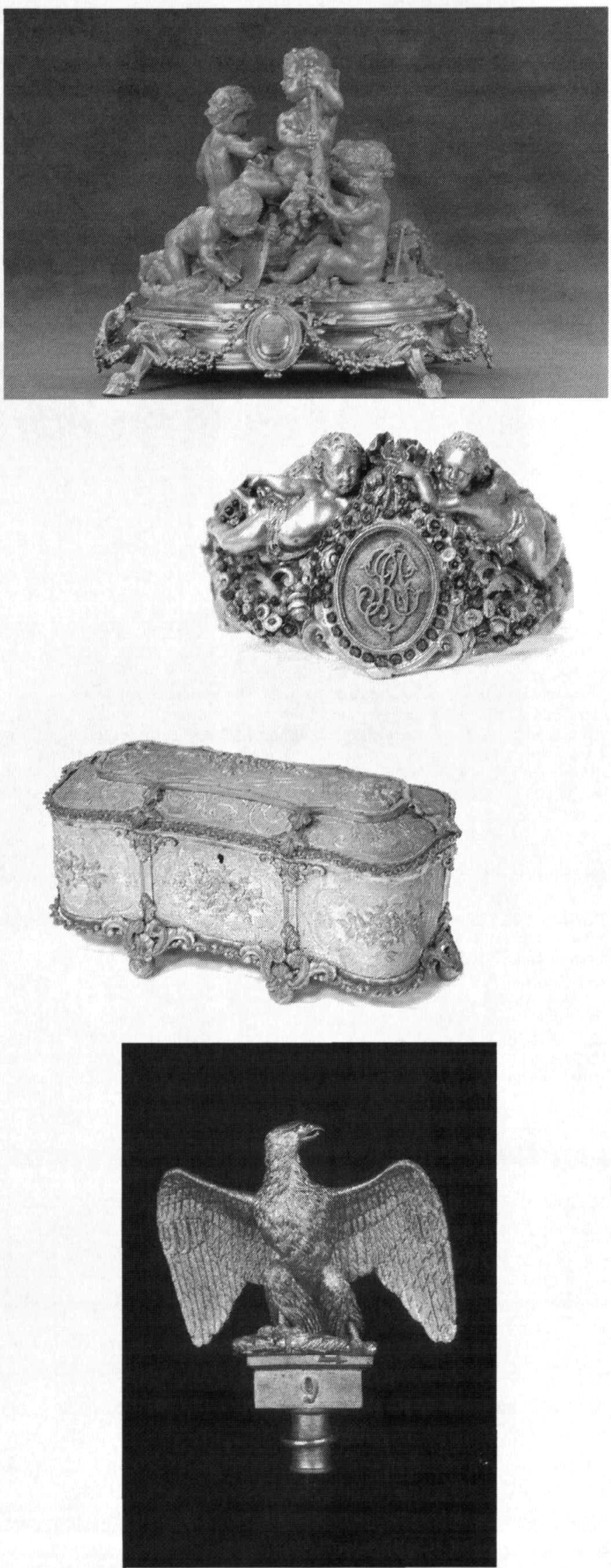

Figure 1 : Certains objets en aluminium (éventuellement associé à d'autres métaux) des collections des Musées Nationaux analysés. De haut en bas et de gauche à droite (voir aussi tableau 1) : fléau de la balance des frères Collot (1855, coll. Arts et Métiers, long. $28 \mathrm{~cm}$, (CC2RMF), Surtout aux Putti (1858, coll. Compiègne, larg. $40 \mathrm{~cm}$, CC2RMF), coupe Dolfuss (1858, coll. Orsay, haut. env. $25 \mathrm{~cm}$, CC2RMF), bracelet (1858, coll. Arts décos, diam. $13,5 \mathrm{~cm}$, CC2RMF), coffret à bijoux (1860, coll. Arts décos, larg. $30 \mathrm{~cm}$, CC2RMF), Hélicoptère (1863, coll. Air et Espace, haut. $50 \mathrm{~cm}$, CC2RMF), Aigle Napoléon III (1861, coll. Armée, haut. 34 cm, CMusée de l’Armée).

Figure 1: Some of the aluminium objects from the French National Museums analysed. See the french legend for the designation of the objects (from top to bottom and left to right). 
abaisser suffisamment le prix de revient et l'aluminium chimique reste un métal semi-précieux, aux débouchés nobles mais limités.

La première application commerciale est la bijouterie, où l'aluminium est d'abord associé à l'or, puis, son prix diminuant, au vermeil et enfin au bronze doré. On essaie le nouveau métal dans beaucoup d'autres petits objets assez luxueux, tels que porte-monnaie, nécessaires de bureau, étuis à cigares, etc. D'autres applications font appel à sa légèreté : appareils scientifiques, jumelles, longues-vues, etc.

Les objets en aluminium chimique qui ont survécu se trouvent aujourd'hui dans les musées et dans les collections privées. L'identification de ces objets - par rapport à ceux qui ont ensuite utilisé le métal « électrolytique »n'est pas facile. L'histoire des objets, leur facture, les poinçons qu'ils portent etc, donnent quelquefois des certitudes, mais, plus souvent, ne permettent que des hypothèses. Nous nous sommes demandé si l'analyse élémentaire pouvait lever certaines des indéterminations. Tel est l'objet de cette étude.
Nous avons effectué l'analyse élémentaire de plus de 100 objets en aluminium, provenant de collections de musées français ou de la collection de l'un des auteurs (tab. 1) au moyen de l'accélérateur AGLAE du Centre de recherche et de restauration des Musées de France. Les principaux résultats obtenus vont être exposés cidessous. Au préalable, les procédés de fabrication de l'aluminium chimique et de l'aluminium électrolytique à ses débuts, ainsi que leur évolution progressive - susceptible d'influer sur la composition du métal - vont être brièvement décrits.

\section{Les procédés de fabrication}

\subsection{Aluminium chimique}

L'aluminium a été isolé pour la première fois par le chimiste Friedrich Wöhler en 1827 , en réduisant le chlorure d'aluminium par le potassium, procédé suggéré - et essayé sans succès - par Oersted en 1825. Le métal fabriqué par Wöhler était impur et contenait du potassium

\begin{tabular}{|c|c|c|c|}
\hline désignation & collection & $n^{\circ}$ inv & datation \\
\hline Fléau balance des frères Collot & $\begin{array}{l}\text { Musée des Arts et } \\
\text { Métiers }\end{array}$ & 162220000 & 1855 \\
\hline Pommeau de canne & $\begin{array}{c}\text { Musée des Arts et } \\
\text { Métiers }\end{array}$ & 370310000 & $1855-1890$ \\
\hline Hélicoptère de Ponton d'Amécourt & $\begin{array}{l}\text { Musée de l'Air et de } \\
\text { l'Espace }\end{array}$ & - & 1863 \\
\hline $\begin{array}{l}\text { Fragment chemise du moteur de l'avion } \\
n^{\circ} 3 \text { de Clément Ader }\end{array}$ & $\begin{array}{l}\text { Musée de l'Air et de } \\
\text { l'Espace }\end{array}$ & - & vers 1897 \\
\hline Coffret à bijoux & Arts décoratifs & 37483 & 1860 \\
\hline Bracelet & Arts décoratifs & 16893 & 1858 \\
\hline Bracelet & Arts décoratifs & 8086 & 1858 \\
\hline Coupe sur pied, Christofle & Musée d'Orsay & DO) $1985-12$ & 1858 \\
\hline $\begin{array}{l}5 \text { aigles de drapeau, arméc de Napoléon } \\
\text { III }\end{array}$ & Musée de l'Armée & $\begin{array}{l}16025, \mathrm{Bd} 276,- \\
187,-239,-236\end{array}$ & 1861 \\
\hline Surtout aux Putti Christofle (base) & $\begin{array}{c}\text { Musée National du } \\
\text { Château de Compiègne }\end{array}$ & FPN3567 & 1858 \\
\hline $\begin{array}{l}20 \text { Médailles et essais monétaires datés } \\
\text { "chimique" }\end{array}$ & JP & - & $1856-1885$ \\
\hline $\begin{array}{l}26 \text { Médailles et essais monétaires datés } \\
\text { "électrolytique" }\end{array}$ & JP & - & $1890-1916$ \\
\hline 8 objets datés, de facture Napoléon III & JP & - & $1855-1890$ \\
\hline $\begin{array}{l}33 \text { objets divers non datés, de facture } \\
\text { Napoléon III }\end{array}$ & JP & - & - \\
\hline $\begin{array}{l}13 \text { objets divers non datés, de facture } \\
\text { fin XIXeme siècle }\end{array}$ & $\mathrm{JP}$ & - & - \\
\hline
\end{tabular}

Tableau 1 : Les objets en aluminium analysés au cours de la présente étude ( 114 objets). Table 1: Aluminium objects analysed (114). 
résiduel, si bien qu'il décomposait l'eau bouillante. C'est Henri Sainte-Claire Deville qui, sur le même principe, a le premier obtenu du métal pur et a déclaré, au vu de ses propriétés, qu'il devait devenir usuel, sous réserve qu'il puisse être produit à bon marché (février 1854). C'est à quoi il s'emploie successivement dans les établissements suivants :

- laboratoire de l'Ecole normale supérieure (1854 1860),

- atelier de la Société de Javel (production du 15 au 29 juin 1855),

- usine des Frères Rousseau, fabricants de produits chimiques à la Glacière, alors dans la banlieue de Paris (mars 1856 - avril 1857),

- usine de Nanterre de la Société Paul Morin et Cie (mi1850 - vers 1865$)$,

- usine de Salindres de la Compagnie des produits chimiques d'Alais et de la Camargue (PCAC) $(1860$ - 1890) (fig. 2).

Les procédés utilisés par Sainte-Claire Deville et la façon dont il les améliore progressivement sont décrits, pour les quatre premiers sites, dans son livre De l'aluminium (Sainte-Claire Deville, 1859), paru en 1859. L'alumine est fabriquée à partir d'alun ammoniacal synthétique du commerce ${ }^{1}$ ou de sulfate d'alumine impur, par calcination. Elle contient du fer comme impureté. On la mélange avec du goudron de houille et c'est par action à chaud du chlore sur ce mélange qu'on obtient le chlorure d'aluminium. Le sodium est obtenu par réduction du carbonate par la houille, en présence de craie qui facilite la circulation du sodium gazeux, dans des récipients en fer. L'aluminium, enfin, résulte de l'action du chlorure d'aluminium, gazeux dès $200^{\circ} \mathrm{C}$, sur le sodium.

A Javel, des récipients en cuivre ayant été utilisés, l'aluminium produit contenait des quantités «notables de ce métal... il avait perdu toute sa ductilité... il avait une teinte grise désagréable ».

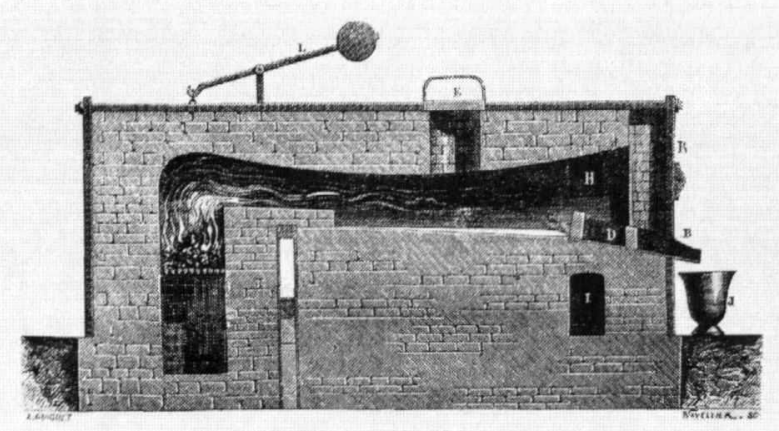

Figure 2: Four à sole pour la fabrication d'aluminium, 1860. On projette par leur regard (E) les produits de la réaction : chlorure double d'aluminium et de sodium, sodium, fluorure de calcium. L'aluminium liquide et la scorie coulent vers le bas de la sole et sont recueillis en (J). Une charge permettait d'obtenir environ $9 \mathrm{~kg}$ d'aluminium (longueur env. $5 \mathrm{~m}$, extrait de Figuier, vers 1867-1869).

Figure 2: Sole furnace for aluminium production, 1860 (circa $5 \mathrm{~m}$. long, after Figuier 1867-1869).
Le procédé évolue à Nanterre, en fonction des résultats de la Glacière: on prépare d'abord du chlorure double d'aluminium et de sodium en introduisant du sel marin en même temps que le charbon et l'alumine dans la cornue où arrive ensuite le chlore. C'est ce chlorure double, solide à la température ambiante et liquide vers $200^{\circ} \mathrm{C}$, sur lequel on fait agir le sodium, en présence de cryolite naturelle $\mathrm{AlF}_{6} \mathrm{Na}_{3}$, qui facilite le rassemblement de l'aluminium liquide. La scorie contient du fluorure d'aluminium que l'on traite pour obtenir de l'alumine réintroduite dans la fabrication. Si la production d'aluminium par réduction de la cryolite par le sodium ne fut pas retenue par Sainte-Claire Deville, en raison du mauvais rendement en aluminium d'une part, et des incertitudes d'approvisionnement d'autre part, le procédé fut utilisé à Amfreville-la-Mi-Voie, près de Rouen, par les frères Tissier, de juillet 1855 au début des années 1860. La cryolite venait du Groenland.

A Salindres, le procédé utilisé est le même qu'à Nanterre, mais la source d'alumine diffère (fig. 2). Dès 1860, celle-ci est produite à partir de bauxite, selon un procédé mis au point par Sainte-Claire Deville et ses collaborateurs : la bauxite est broyée, mélangée avec du carbonate de soude, chauffée à haute température. Il en résulte de l'aluminate de soude, qui est dissous dans l'eau. L'oxyde de fer et le silico-aluminate, insolubles, sont éliminés par décantation et filtration. Le liquide est soumis à un courant de $\mathrm{CO}_{2}$ qui déplace l'alumine et régénère le carbonate de soude. D'après Lejeal (Lejeal, 1894), un peu d'oxyde de fer est précipité avec l'alumine. La fabrication de l'aluminium se poursuit à Salindres jusqu'en 1890, date à laquelle elle est arrêtée par suite de l'arrivée sur le marché de métal électrolytique à bas coût, produit à proximité des chutes d'eau génératrices de l'énergie électrique nécessaire.

\subsection{Aluminium électrolytique}

Rappelons que le procédé de fabrication d'aluminium, breveté simultanément par Héroult en France et par Hall aux Etats-Unis consiste à électrolyser de l'alumine en solution dans un bain de cryolite, vers $900^{\circ} \mathrm{C}$. La Société électrométallurgique française (SEMF), commença, en avril 1889, à fabriquer à Froges (fig. 3) des alliages d'aluminium (bronze et ferro-alliages) par ce procédé, puis, à partir de septembre 1889, de l'aluminium pur. L'alumine utilisée venait de Silésie, la cryolite du Danemark. L'usine de La Praz (1893) utilise à ses débuts les mêmes sources de matières premières. L'alumine importée était fabriquée à partir de bauxites françaises «blanches" (Régnier, 1989) sans doute pauvres en fer, et le procédé de fabrication utilisait la voie ignée de Deville.

L'usine d'alumine de Gardanne, exploitant le procédé Bayer, construite par la Société française de l'alumine pure en 1893-1894, fut aussitôt acquise par la SEMF qui alimenta ses usines à partir de là au fur et à mesure de la mise au point de la production qui dura plusieurs années. La bauxite broyée est attaquée par de la soude

${ }^{1}\left(\mathrm{NH}_{4} \mathrm{Al}\left(\mathrm{SO}_{4}\right)_{2}-12\left(\mathrm{H}_{2} \mathrm{O}\right)\right.$. Peut être préparé notamment à partir de l'action de l'acide sulfurique sur des argiles ou par grillage de schistes alumineux. 


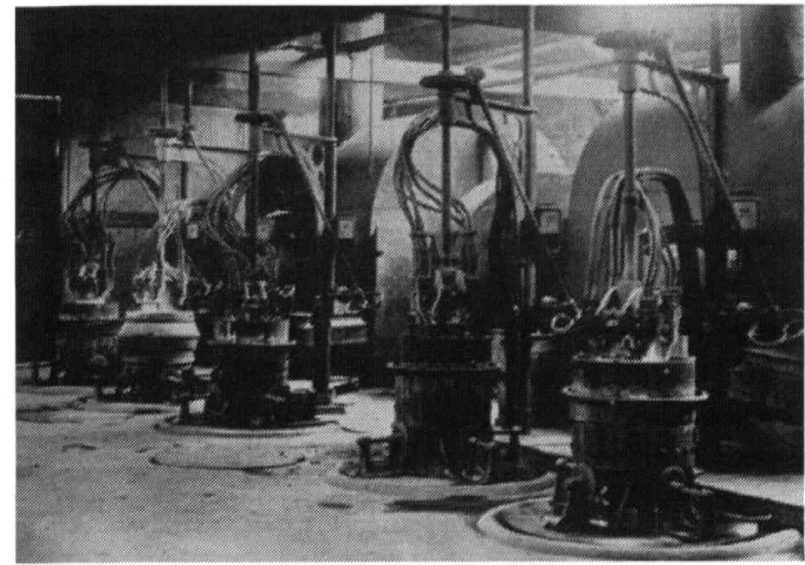

Figure 3 : Atelier de la Société Electrométallurgique de Froges, env. 1889. Il comporte 6 cuves que l'on faisait tourner pour casser les croûtes du bain et faciliter la dissolution de l'alumine (C) Institut pour l'Histoire de l'Aluminium).

Figure 3: Workshop of the Société Electrométallurgique de Froges, France, circa. 1889.

caustique à $250^{\circ} \mathrm{C}$ sous pression dans des autoclaves. La liqueur obtenue est décantée, puis on y fait précipiter à froid l'alumine.

PCAC se réintroduit dans l'industrie de l'aluminium en 1897, par le rachat de l'usine de Calypso, créée par les Frères Bernard et qui, après avoir échoué sur les procédés Minet $^{2}$, exploitait le procédé Hall. En 1914, l'usine passe au procédé Héroult. Elle est alimentée en alumine par Salindres, c'est-à-dire par le procédé Deville dès 1898 et jusqu'en 1906, puis par le procédé Bayer.

En 1904, PCAC commence la construction d'une usine d'électrolyse à St-Jean-de-Maurienne. En 1908, cinq sociétés produisent de l'aluminium en France. Elles utilisent toutes le même procédé et créent en 1911 un comptoir de vente commun, l'Aluminium français.

\section{Méthode d'analyse}

\subsection{Choix du corpus}

114 objets ont été analysés. Parmi eux, figurent la plupart des objets des débuts de l'aluminium que possèdent les musées français (fig. 1 et tab. 1) :

- Les deux bracelets ciselés par Honoré Bourdoncle (vers 1858) et un grand coffret à bijoux des Arts Décoratifs (1860).

- Le fléau de la balance des Frères Collot, qui figurait à l'exposition universelle de 1855 , et un pommeau de canne du Musée des Arts et Métiers.

- La coupe sur pied en aluminium et or du Musée d'Orsay, réalisée en 1858 par Christofle pour Emile Dolfuss, président de la Sociéte industrielle de Mulhouse.
- Le groupe de Putti offert par Christofle à Napoléon III, «pour l'aide et les encouragements qu'il apporta aux travaux consacrés par le savant Henri Sainte-Claire Deville à la fabrication de l'aluminium » (1858) (Musée National du Château de Compiègne).

- Cinq aigles en aluminium doré, du Musée de l'Armée (1861).

- L'hélicoptère à vapeur de Ponton d'Amécourt (1863), qui n'a jamais volé malgré l'utilisation d'aluminium ; un fragment de chemise du moteur de l'avion $\mathrm{n}^{\circ} 3$ de Clément Ader (1897) (Musée de 1'air et de 1'espace).

La plupart de ces objets ont une origine et une histoire parfaitement connues.

Les autres objets analysés proviennent de la collection de l'un des auteurs (collection JP, fig. 4). Ils concernent près de 50 médailles datées ou essais monétaires ${ }^{3}$ dont une moitié est antérieure à 1890 ; pour l'autre moitié, les dates s'échelonnent entre 1890 et 1916 . S'y ajoutent une trentaine de bijoux et une vingtaine d'objets divers, la plupart de facture Napoléon III.

Les objets analysés appartiennent à quatre catégories :

1) ceux pour lesquels l'appartenance à la période de l'aluminium chimique est à peu près certaine. Il s'agit des objets des musées dont l'histoire est connue, de médailles de la période considérée, de bijoux portant un poinçon d'orfèvre identifié et dont les dates d'insculpation ${ }^{4}$ et de biffage sont connues. Au total, 41 pièces.

2) les objets en aluminium électrolytique, au nombre de 26 : médailles et essais monétaires postérieurs à 1889 , et pièce de l'appareil de C. Ader.

3) des objets dont la facture correspond à ce qui se faisait de 1850 à 1890 , notamment des bijoux en aluminium ciselé (33 objets).

4) des objets divers dont on souhaiterait savoir de quand ils datent et de quel côté ils se placent, et pour lesquels existe un réel doute (13 objets).

\subsection{Conditions d'analyse}

Les petites dimensions de la plupart des objets rendaient le prélèvement souvent délicat voire impossible, une technique d'analyse en surface avec une bonne résolution surfacique était par conséquent nécessaire, sachant qu'une bonne sensibilité sur un maximum d'éléments était également requise (de l'ordre de la centaine de ppm). Nous avons opté pour la technique PIXE protons (Particule Induced X-ray Emission) qui, telle qu'elle est pratiquée sur l'accélérateur AGLAE du Centre de Recherche et de Restauration des Musées de France, permet d'accéder aux conditions voulues. Les spectres PIGE protons (Particule Induced Gamma-ray Emission) ont été systématiquement enregistrés pour tenter de traquer $\mathrm{Na}, \mathrm{Mg}$ et $\mathrm{Si}$, sans succès (notamment à cause des

\footnotetext{
${ }^{2}$ Minet conduisait l'électrolyse à plus basse température que Héroult, dans des bains complexes, par exemple dans un mélange de cryolite et de chlorure de sodium.

${ }^{3}$ Avant de mettre en circulation une nouvelle pièce de monnaie, la Monnaie effectue des essais, appelés essais monétaires, qui portent aussi bien sur la gravure, sur le métal que sur les dimensions de la pièce. Le nombre de pièces frappées dans ces essais est assez important, ce qui permet de juger du comportement au matriçage, de la résistance à l'usure, etc.. Elles sont ensuite vendues aux collectionneurs.

${ }^{4}$ Action de marquer un objet métallique avec un poinçon. La date d'insculpation est celle à laquelle le poinçon d'un orfêvre a été insculpé dans une plaque du bureau des Garanties, et enregistré par ce bureau.
} 

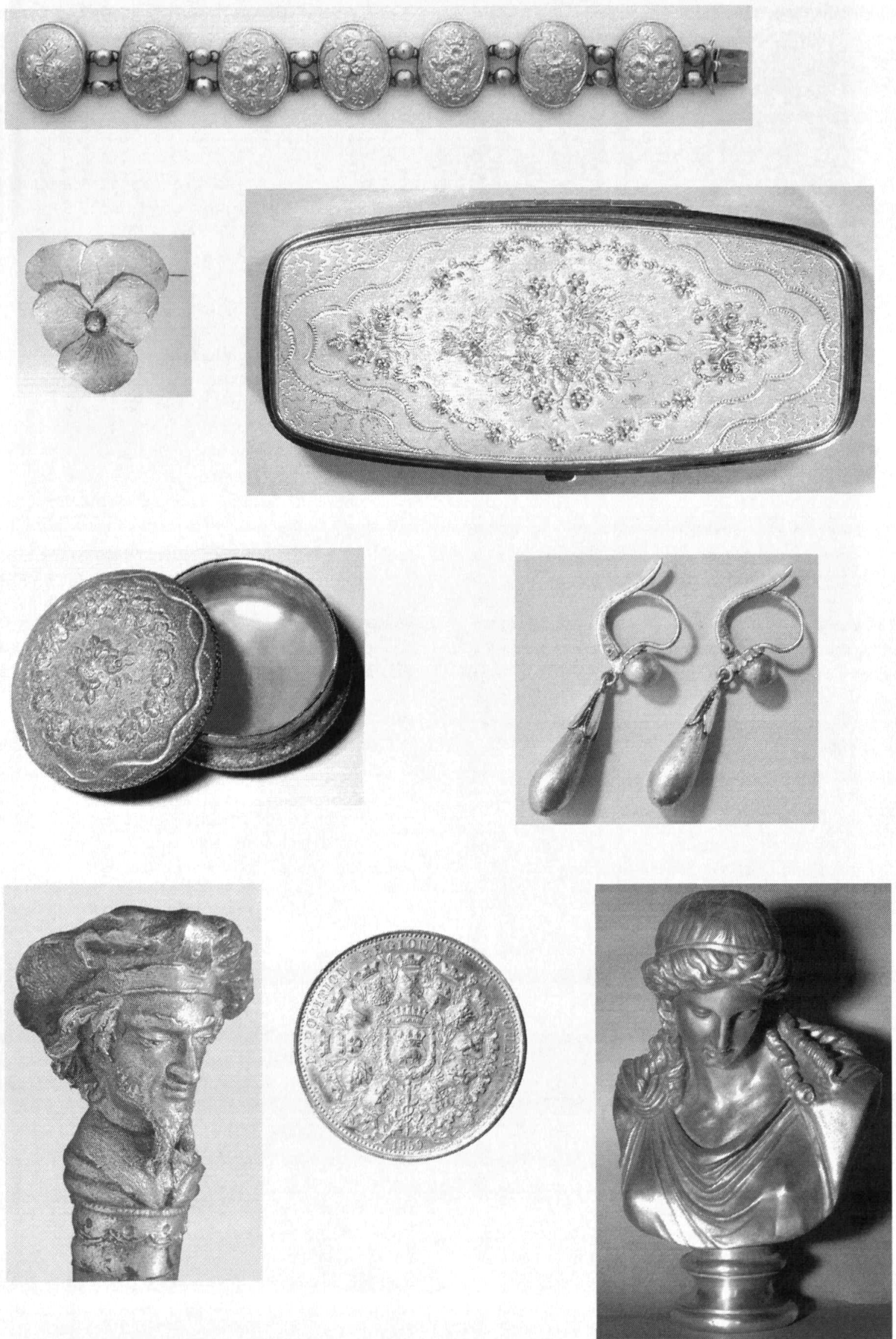

Figure 4 : Certains objets en aluminium de facture Napoléon III, de la collection JP (CPlateau). De haut en bas et de gauche à droite : bracelet (inv. 13903 , long. $18 \mathrm{~cm}$ ), broche (inv. 9967, diam. $4 \mathrm{~cm}$ ), étui à cigares (inv. 7207, long. $14 \mathrm{~cm}$ ), boîte à pilules (inv. 11873, diam. $5 \mathrm{~cm}$ ), boucles d'oreille (inv. 11778, haut. $4 \mathrm{~cm}$ ), pommeau de canne Méphisto (inv. 12266, haut. $7 \mathrm{~cm}$ ), médaille de Rouen (1859, inv. 15241, diam. $4 \mathrm{~cm}$ ), buste de Eiréné (vers 1862, inv. 15331, haut. $32 \mathrm{~cm}$ ).

Figure 4: Some of the aluminium objects exhibiting a Napoléon III typology, private collection (JP). 
interférences avec $\mathrm{Al}$ ). Le faisceau de protons de $3 \mathrm{MeV}$ présente, après extraction à l'air, un diamètre d'environ $50 \mu \mathrm{m}$; une homogénéisation de la mesure est réalisée par balayage de la surface (environ $1 \mathrm{x} 1 \mathrm{~mm}$ ) ; la profondeur analysée est d'environ $50 \mu \mathrm{m}$. Deux détecteurs SiLi sont utilisés pour le PIXE : un est consacré aux basses énergies avec balayage de $\mathrm{He}$, l'autre est dédié aux plus hautes énergies avec un filtre de $50 \mu \mathrm{m}$ de Al. Pour plus de détails sur la technique, voir Calligaro et al., 1998 et Dran et al., 2000.

Pour la grande majorité des cas, les analyses ont été conduites à même l'objet après un léger nettoyage à l'acétone à l'endroit de la mesure. Pour certaines pièces volumineuses de la collection Jean Plateau, des prélèvements ont été effectués (micro perceuse, mèche de $1 \mathrm{~mm}$ en acier). Les copeaux recueillis ont été mis sous forme de pastilles de $5 \mathrm{~mm}$ de diamètre (pression 2 bars), qui ont été analysées comme les objets. 23 éléments ont été analysés ${ }^{5}$, quatre matériaux certifiés de référence ont permis de caractériser la fiabilité des mesures.

\section{Résultats}

\subsection{Les éléments discriminants}

Ce sont les résultats relatifs aux objets bien datés (deux premières calégories) qui doivent permettre de distinguer métal chimique et métal électrolytique. Nous avons recherché les éléments dont le dosage mettait en évidence des valeurs différentes selon l'époque (avant ou après 1890) à laquelle l'objet était supposé avoir été fabriqué. Sur les 22 éléments dosés (autres que $\mathrm{Al}$ ), trois apparaissent discriminants (fig. 5) : le fer, le manganèse et le plomb. On voit en effet que, à quelques exceptions près, les échantillons obtenus avant 1890 présentent des taux systématiquement supérieurs aux valeurs mesurées dans les aluminiums élaborés après 1890. Les valeurs limites sont (\% massique) :

$\mathrm{Fe}=0,75 \% \quad \mathrm{Mn}=0,013 \% \quad \mathrm{~Pb}=0,015 \%$.

De plus, trois autres éléments affichent une tendance analogue à la précédente, à savoir des teneurs supérieures dans les aluminiums chimiques : le chrome, le zirconium et l'argent (fig. 5). Cependant, leur pouvoir discriminant est nettement moins important que celui des éléments Fe, $\mathrm{Mn}, \mathrm{Pb}$ (voir paragraphe suivant). Les inégalités suivantes ne sont respectées qu'avant 1890 :

$\mathrm{Cr}>0,015 \% \quad \mathrm{Zr}>0,004 \% \quad \mathrm{Ag}>0,006 \%$

\subsection{Quelques exceptions à la règle}

On remarque sur la figure 5 que plusieurs objets affichent des teneurs en l'un ou plusieurs des six éléments discriminants qui contredisent leur datation dans l'une ou l'autre période. Concernant les trois éléments $\mathrm{Cr}, \mathrm{Zr}$ et $\mathrm{Ag}$, le nombre d'anomalies est important. Cependant il est difficile de juger de la réalité de ce phénomène, étant donné la faiblesse des teneurs limites en regard des per- formances de la mesure. En particulier, la proximité des teneurs limites et des limites de quantification (estimées à au moins trois fois les limites de détection) peut être à l'origine de l'important recouvrement constaté entre les deux périodes, et rend, quoi qu'il en soit, la discrimination peu fiable dans de nombreux cas.

Par conséquent, nous tiendrons peu compte dans ce qui suit des éléments $\mathrm{Cr}, \mathrm{Zr}$ et $\mathrm{Ag}$, pour nous intéresser principalement aux teneurs en $\mathrm{Fe}, \mathrm{Mn}$, et $\mathrm{Pb}$.

\subsubsection{Première période}

Pour le fer, on remarque trois anomalies. Un essai monétaire, l'essai au Ballon (1870) se démarque particulièrement, puisque en plus du fer les deux teneurs en $\mathrm{Mn}$ et $\mathrm{Pb}$ se situent également sous les limites. On remarquera qu'il en est de même pour les trois éléments $\mathrm{Cr}, \mathrm{Zr}$ et $\mathrm{Ag}$. D'autre part, un autre essai monétaire, de Merley (1888) affiche également une faible teneur en $\mathrm{Fe}$, ainsi qu'en $\mathrm{Cr}, \mathrm{Zr}$ et Ag. Enfin, sur trois dosages effectués parmi les différentes pièces assemblées du Surtout aux Putti de Christofle de 1858 (deux putti, et la base sur laquelle sont assemblés les putti), les deux putti donnent des valeurs en Fe faibles $(0,6 \%$ contre $2 \%$ pour la base).

Pour le manganèse, on observe aussi trois anomalies. L'essai monétaire au Ballon cité plus haut, la médaille de l'exposition de Rouen de 1859 (noter aussi les anomalies pour le $\mathrm{Pb}$, et pour le $\mathrm{Cr}, \mathrm{Zr}, \mathrm{Ag}$ ), et la médaille du jubilé de François-Joseph d'Autriche (1873) (qui affiche en sus une faible teneur en $\mathrm{Cr}$ ).

Pour le plomb, on relève deux valeurs faibles dans des objets cités ci-dessus : l'essai monétaire au Ballon et la médaille de Rouen.

\subsubsection{Deuxième période}

On note une exception pour le fer (médaille de l'exposition de Bruxelles, 1897), et deux pour le plomb (médaille de la Société Electrométallurgique de Froges, 1893, et la chemise du moteur de l'avion n 3 de Clément Ader, 1897) qui n'appellent pas de commentaires particuliers. Pour le manganèse, une médaille et deux essais monétaires des Produits Chimiques d'Alais et de la Camargue (PCAC) en 1908 en comportent environ $0,4 \%$. On remarquera que ces trois objets sont également marqués par des teneurs en argent relativement importantes (autour de $0,5 \%$ en masse). Signalons enfin que la médaille de Purrey (1910) présente une teneur élevée en $\operatorname{Zr}(0,02 \%)$.

\subsection{Les autres éléments}

Pour tout discriminants qu'ils soient, les six éléments $\mathrm{Fe}, \mathrm{Mn}, \mathrm{Pb}, \mathrm{Zr}$ et $\mathrm{Cr}, \mathrm{Ag}$ ne sont pas les seules impuretés mesurées. Le silicium en particulier est présent dans quasiment tous les objets analysés, à des teneurs souvent supérieures à $1 \%$ en masse et pouvant dépasser $10 \%$. Cependant, la variation observée ne semble pas être corrélée à l'un ou l'autre procédé d'élaboration. On remarque

\footnotetext{
${ }^{5} \mathrm{Al}, \mathrm{Si}, \mathrm{S}, \mathrm{Ti}, \mathrm{V}, \mathrm{Cr}, \mathrm{Mn}, \mathrm{Fc}, \mathrm{Co}, \mathrm{Ni}, \mathrm{Cu}, \mathrm{Zn}, \mathrm{Ga}, \mathrm{As}, \mathrm{Se}, \mathrm{Zr}, \mathrm{Ag}, \mathrm{Cd}, \mathrm{Sn}, \mathrm{Sb}, \mathrm{Au}, \mathrm{Pb}, \mathrm{Bi}$
} 

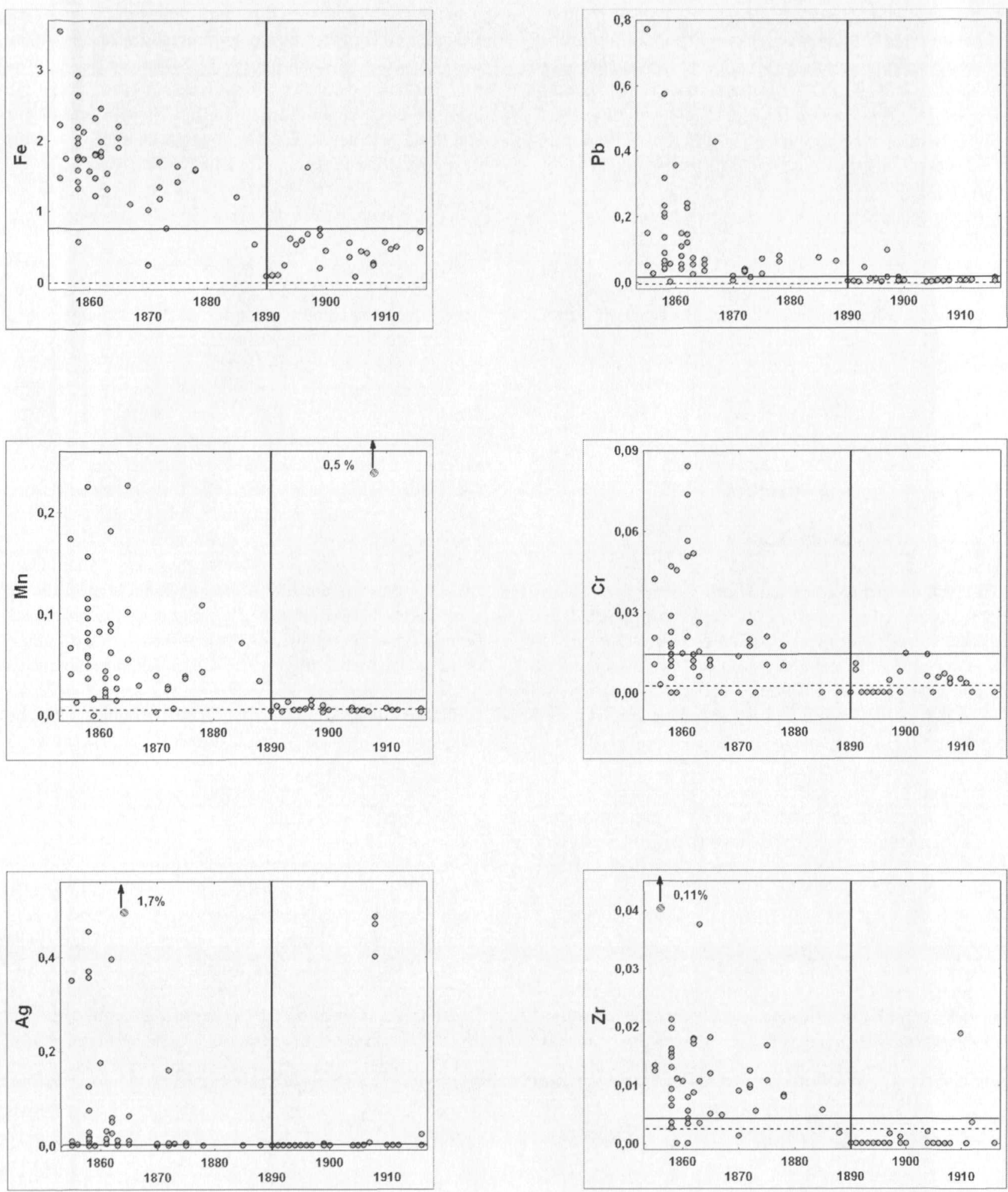

Figure 5 : Teneurs en $\mathrm{Fe}, \mathrm{Mn}, \mathrm{Pb}, \mathrm{Zr}, \mathrm{Cr}, \mathrm{Ag}$ (\% massique) des objets en aluminium obtenues par PIXE, en fonction de leur date de fabrication. Le trait horizontal en pointillés indique la limite de détection. Le trait vertical en gras marque la date de 1890 , c'est-à-dire la date de transition entre la période de l'aluminium chimique et celle de l'aluminium électrolytique. Les graphiques montrent clairement qu'à partir de 1890, les teneurs en $\mathrm{Fe}, \mathrm{Mn}, \mathrm{Pb}, \mathrm{Zr}$, $\mathrm{Cr}, \mathrm{Ag}$ sont systématiquement plus faibles. Le trait horizontal en gras indique la teneur limite choisie pour discriminer les 2 périodes. L'erreur totale sur la mesure est estimée à $10 \%$.

Figure 5: $\mathrm{Fe}, \mathrm{Mn}, \mathrm{Pb}, \mathrm{Zr}, \mathrm{Cr}, \mathrm{Ag}$ contents (wt \%) in the aluminium objects, sorted according to their date of fabrication (PIXE measurements). The horizontal dash line indicates the detection limit. The vertical bold line indicates year 1890, when the chemical process started being replaced by the electrolytic process. The graphs show clearly that from 1890 onwards, the $\mathrm{Fe}, \mathrm{Mn}, \mathrm{Pb}, \mathrm{Zr}, \mathrm{Cr}, \mathrm{Ag}$ contents are systematically lower. The horizontal bold line shows the content level which allows to discriminate between the 2 periods. Global measurement error is estimated at $10 \%$. 
également des teneurs atypiques en certains éléments dans quelques objets isolés. Citons notamment le cuivre, dont les teneurs sont systématiquement supérieures à $2 \%$ dans les Aigles, atteignent près de $3 \%$ pour la soupape de l'Hélicoptère, et dépassent $4 \%$ dans la partie principale du fléau de la balance des frères Collot. Que ces éléments soient volontairement ajoutés ou non (il n'est pas toujours facile de trancher), ces teneurs extrêmes ne permettent pas non plus de discriminer un procédé d'élaboration.

On fera part au passage de notre déception concernant deux médailles des usines du Partinium. En effet, le nom de Partinium recouvrait beaucoup de compositions différentes, que M. Partin tenait soigneusement confidentielles et dont on ne sait pas comment il les utilisait ! Ces alliages étaient notamment appliqués à la construction automobile. L'un d'eux constitue en particulier la coque fuselée de la voiture électrique la « Jamais contente » qui, le $1^{\text {er }}$ mai 1899 a atteint la vitesse de $105 \mathrm{~km} / \mathrm{h}$. Nous espérions par l'analyse des deux médailles en notre possession mettre au jour une ou plusieurs compositions mystérieuses. Or, si les compositions des deux médailles sont très différentes, ce que confirment les résultats d'analyses de Partinium trouvées dans la littérature (Hammer, 2005), aucune composition atypique n'a été mise au jour. Ainsi, l'une des médailles ne présente aucun élément apparemment ajouté, l'autre $4 \%$ de $\mathrm{Cu}$ qui sont communs à plusieurs objets analysés ici (notamment un des premiers objets en aluminium, cité ci-dessus, le fléau de la balance des frères Collot); les spectres d'impuretés sont analogues à la période électrolytique.

\section{Discussion}

\subsection{Définition d'un critère}

S'il apparaît que les teneurs en $\mathrm{Fe}, \mathrm{Mn}$, et $\mathrm{Pb}$ et, dans une moindre mesure $\mathrm{Cr}, \mathrm{Zr}$ et $\mathrm{Ag}$ sont susceptibles de donner des indications sur l'origine - chimique ou électrolytique - d'un échantillon d'aluminium, il apparaît aussi que l'application des critères précédents ne peut pas conduire à une certitude. Ceci pour plusieurs raisons. D'abord, des aluminiums de l'une et de l'autre origine peuvent présenter des teneurs en impuretés très proches, voire égales aux limites indiquées ci-dessus ; il n'est donc pas exclu qu'il puisse y avoir recouvrement dans certains cas (fig. 5). Ensuite, il pourrait exister du métal d'origine chimique dont nous ignorons la composition. L'exemple de la médaille de Rouen (1859) incite à la prudence. Il s'agit du seul objet de la période « chimique » pour lequel deux des éléments examinés, $\mathrm{Mn}$ et $\mathrm{Pb}$, ont des teneurs inférieures aux limites indiquées ci-dessus (hormis l'essai monétaire au Ballon avec ses basses teneurs en Mn, $\mathrm{Fe}$ et $\mathrm{Pb}$, qui constitue un cas particulier, voir ci-après). Or, on est à l'époque où l'on produisait de l'aluminium d'une part à Nanterre et d'autre part, en moindre quantité, à Amfreville-la-Mi-Voie, près de Rouen, à partir de cryolite. Il est vraisemblable que pour la médaille de l'exposition de Rouen, on ait employé le métal fabriqué sur place et que les différences de procédés expliquent les différences de composition. On peut noter de plus que la teneur en fer, relativement élevée, $2,1 \%$, est compatible avec la présence de fer comme principale impureté dans la cryolite du Groenland utilisée.

Le cas de l'essai monétaire au Ballon mérite un commentaire (fig. 6). L'avers comporte un ballon rond, seul moyen de communication entre Paris et le reste du pays pendant le siège de 1870-1871 et symbole de l'unité nationale, ainsi que la mention : «Gouvernement de la défense Nationale ». Au revers « République française » et « $1870 »$. C'est la date de la réalisation de la matrice. Cette année-là, et dans les années qui ont suivi, des essais de frappe avec cette matrice ont été réalisés en or, en argent, en maillechort et en aluminium (Mazard, 1969 et Schmitt, 2005). Il est donc tout à fait possible que l'essai en aluminium que nous avons ici ait été frappé bien après 1870 , et même après $1889-90$ : la composition caractéristique de l'aluminium électrolytique mise en évidence opterait pour cette datation tardive. Une information intéressante qu'apporte l'analyse élémentaire. Il en est probablement de même pour l'essai monétaire de Merley.

De plus, si à Salindres le procédé était relativement stable, pendant toute la période précédente (avant 1860) les producteurs ont fait des quantités importantes d'essais, avec notamment des tentatives nombreuses de purification du métal. On le remarque notamment sur l'exception signalée plus haut du surtout aux Putti qui, au sein d'un même objet, montre des variations importantes sur le fer avec en particulier un passage sous la teneur limite. De la même façon, les trois parties analysées du fléau de la balance des Frères Collot montrent une grande dispersion dans leur composition, avec en particulier la présence importante de $\mathrm{Cu}$ et de $\mathrm{Zr}$ dans la partie principale du fléau de la balance que l'on ne retrouve ni dans la réglette ni dans l'aiguille (fig. 1). D'ailleurs, même la production de Salindres a fait l'objet d'essais, comme en témoigne par exemple la teneur inhabituelle en $\mathrm{Ni}(1,4 \%)$ remarquée sur la Médaille au Gardon (1872).

Enfin, la présence d'éléments d'addition ou d'impuretés accidentelles à teneurs élevées peut rendre caduques les critères de discrimination énoncés plus haut. Soit parce

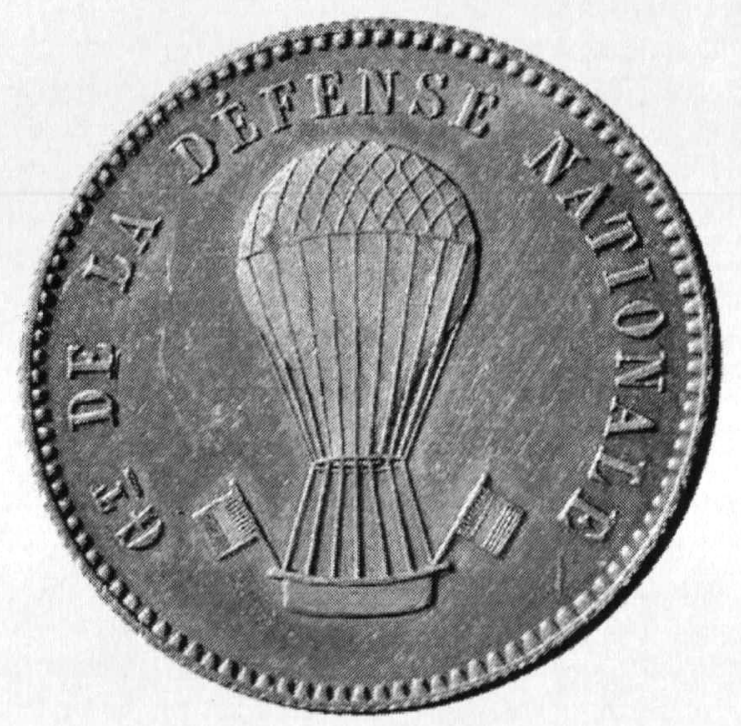

Figure 6 : Essai monétaire au Ballon (marqué 1870, coll. JP inv. 12076, diam. $3 \mathrm{~cm}$ ) CPlateau.

Figure 6: Die-test coin " Ballon ». 
que l'un des éléments discriminants est volontairement ajouté. C'est le cas pour le Mn dans les essais monétaires de $\mathrm{PCAC}$, où il s'agit manifestement d'un élément d'alliage destiné à améliorer les propriétés du métal. Soit parce que l'élément ajouté introduit à sa suite un cortège d'impuretés qui lui est propre. Ainsi, il est possible que ce soit des cuivres d'origines différentes qui aient été ajoutés dans les Aigles de Napoléon III. Ceci expliquerait les variations de teneurs observées notamment sur $\mathrm{Ag}$ et $\mathrm{Pb}$, alors que ces objets ont été fabriqués dans un intervalle de temps relativement restreint. En effet, la fabrication des aigles s'est déroulée au début des années 1860 , c'està-dire dans les premières années de l'Usine de Salindres. A cette époque, on estime une production de $700 \mathrm{~kg} / \mathrm{an}$. La fabrication des 217 aigles représente environ $200 \mathrm{~kg}$, soit environ $1 / 3$ de la production annuelle.

Finalement, pour différencier les deux âges de l'aluminium, il convient de considérer simultanément les teneurs des trois éléments $\mathrm{Fe}, \mathrm{Mn}, \mathrm{Pb}$, et d'appliquer avec prudence les règles suivantes, que suggèrent les résultats qui précèdent

1) si deux des trois critères $\mathrm{Fe}>0,75 \%, \mathrm{Mn}>0,013 \%$ et $\mathrm{Pb}>0,015 \%$ sont remplis, l'origine chimique de l'aluminium correspondant est très probable. La probabilité sera d'autant plus grande que les teneurs en l'un des trois autres éléments sera grande $(\mathrm{Cr}>0,015 \%, \mathrm{Zr}$ $>0,004 \%$, ou Ag > 0,006\%).

2) sinon, le métal a de fortes chances d'avoir été obtenu par électrolyse.

\subsection{Origine des différences de composition}

Les teneurs en fer relativement élevées dans l'aluminium chimique s'expliquent bien. En effet, le fer donne un chlorure $\mathrm{FeCl}_{3}$ qui est miscible en toutes proportions, à l'état solide comme à l'état liquide, avec $\mathrm{AlCl}_{3}$. C'est un des problèmes qu'avait rencontré ALCOA (années 1950-1960), pour son procédé de fabrication électrolytique d'aluminium par la voie des chlorures (Leroy, 2005). Par contre, nous ignorons l'origine des autres impuretés dans l'aluminium chimique.

D'une manière plus générale, les brèves données qui décrivent en première partie de cet article les différents procédés d'élaboration, procédés chimiques et procédé par électrolyse montrent que, au-delà de la distinction entre ces deux procédés, les choses sont beaucoup trop complexes pour que l'on puisse tracer la filiation qui relie le minerai à l'objet fini par sa composition, et inversement. Encore n'avons-nous pas mentionné la diversité des sources de matières premières, et par exemple de la bauxite.

\subsection{Application des critères}

Voyons maintenant à quoi conduit l'application des règles ci-dessus aux objets non datés. Les graphes de la figure 7 représentent les proportions des six éléments discriminants dans ces objets. Nous avons distingué en début d'article deux catégories pour ce type d'objets :
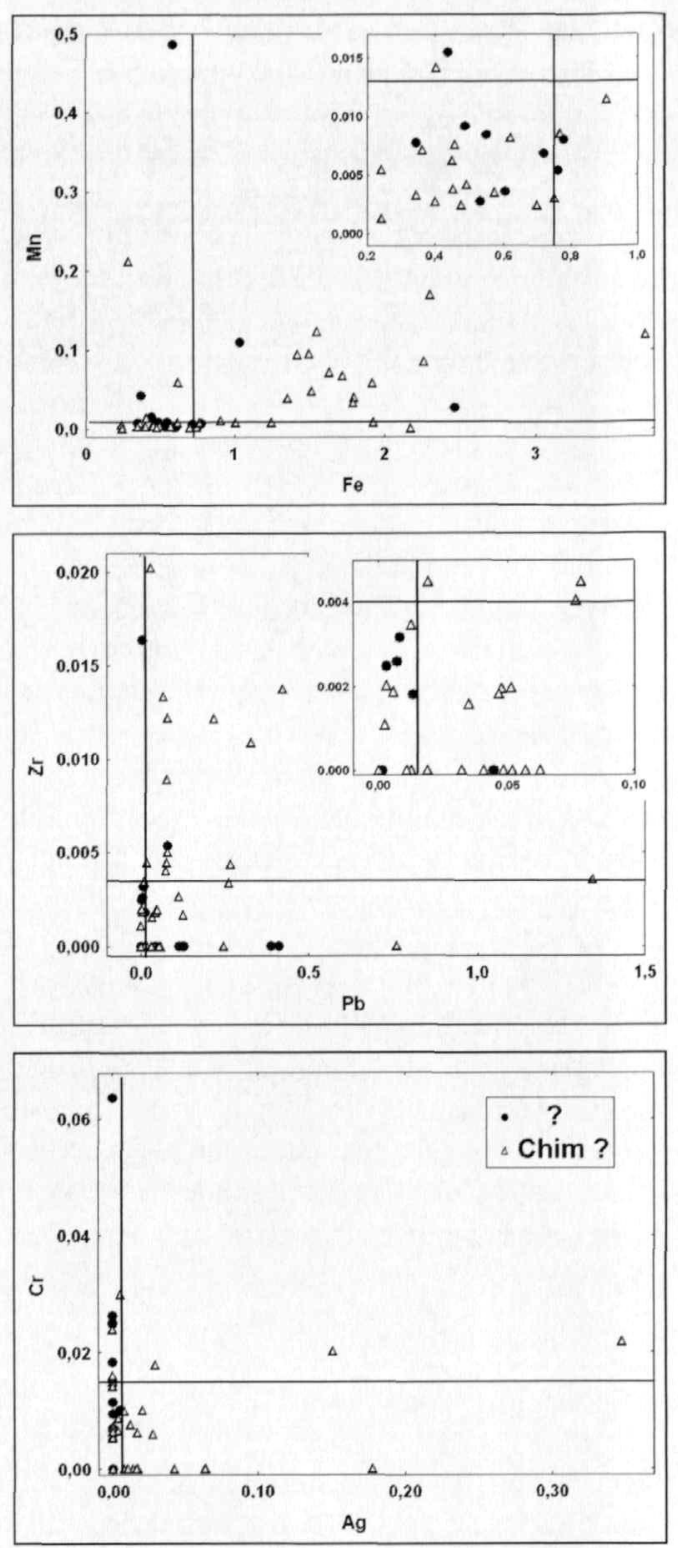

Figure 7 : Teneurs en $\mathrm{Fe}, \mathrm{Mn}, \mathrm{Pb}, \mathrm{Zr}, \mathrm{Cr}$ et $\mathrm{Ag}$ (\% massique) des objets en aluminium non datés, en fonction de leur catégorie. La légende apparaît dans le diagramme $\mathrm{Ag} / \mathrm{Cr}$ : les triangles vides représentent la catégorie 3 (objets de typologie «chimique»), les points noirs représentent les objets de la catégorie 4 (sans typologie marquée). Les barres horizontales et verticales en gras indiquent les teneurs limites discriminant la période (voir figure 5).

Figure 7: $\mathrm{Fe}, \mathrm{Mn}, \mathrm{Pb}, \mathrm{Zr}, \mathrm{Cr}, \mathrm{Ag}$ contents (wt \%) in the non-dated aluminium objects, sorted according to their category. The legend is to be seen on the diagram $\mathrm{Ag} / \mathrm{Cr}$. Horizontal and vertical bold lines indicate the discriminating content limits (see figure 5).

- Catégorie 3 : Il s'agit de 33 objets dont l'histoire et la facture (aluminium ciselé, style Napoléon III, décor rocaille, néo Louis XV...) suggèrent qu'ils pourraient être en aluminium chimique. Les règles précédentes donnent 18 objets sur 33 en aluminium chimique (ils remplissent tous le critère sur $\mathrm{Fe}$ et $\mathrm{Pb}$ ). Les 15 objets restant peuvent très bien avoir été faits après 1890 , en réalisant avec du métal électrolytique les mêmes objets que l'on faisait auparavant en métal chimique. C'est certainement le cas pour certains d'entre eux. Il sera cependant difficile de faire admettre que le coffret à bijoux du Musée des arts 
décoratifs, dont la composition est celle de métal électrolytique, ne soit pas né vers 1860 . Il se pourrait aussi que certains aient été fabriqués avec un métal chimique de composition particulière, que nous ignorons. Ainsi, l'étui à cigares en dépôt au Musée des arts et métiers a une composition très semblable à celle de la médaille de Rouen, dont nous avons dit plus haut qu'elle pourrait avoir été faite avec du métal d'Amfreville-la-Mi-Voie, élaboré à partir de cryolite : pour les deux objets, la teneur en fer est voisine de $2 \%$, et de plus, les teneurs en $\mathrm{Mn}, \mathrm{Pb}$, et aussi en $\mathrm{Ti}, \mathrm{V}, \mathrm{Cr}, \mathrm{Zr}, \mathrm{Ag}$ sont inférieures aux limites de détection.

- Catégorie 4 : Il s'agit de 13 objets divers dont la facture correspond à ce qui pouvait se faire à la fin du XIXème siècle. Il existe pour eux un réel doute, et on souhaiterait savoir de quand ils datent et de quel côté ils se placent. D'après les critères, 4 objets sont en aluminium chimique. Les autres sont donc très probablement en métal électrolytique. Ce sont là des informations intéressantes qui, à l'heure actuelle, découlent exclusivement des critères mis en place.

\section{Conclusions}

Le dosage du fer, du manganèse et du plomb dans l'aluminium fournit des informations sur l'origine du métal, chimique ou électrolytique. Le principal résultat obtenu ici est que, pour un aluminium relativement pur, si les teneurs en ces éléments répondent à 2 des 3 critères :

$\mathrm{Fe}>0,75 \% \quad \mathrm{Mn}>0,013 \% \quad \mathrm{~Pb}>0,015$ $\%$, l'origine chimique est à peu près certaine. De fortes teneurs en chrome, zirconium et/ou argent renforcent la probabilité de l'origine chimique.

Une exception est apparue cependant avec la médaille de Rouen, qui laisse ouverte la possibilité de l'existence d'un métal chimique encore sous représenté dans le corpus analysé ici, et qu'il n'a pas été possible par conséquent de caractériser. Ainsi, si l'application de ces critères à des objets non datés mais de typologie « chimique » a mis en lumière des erreurs probables d'attribution, des doutes subsistent pour certains objets : ces derniers, dont la composition correspond à celle du métal électrolytique, pourraient cependant avoir été réalisés avec un métal chimique que nous ignorons.

Pour préciser davantage, différentes pistes sont à envisager. D'une part le présent corpus doit être complété, à la fois par l'analyse d'autres objets et par le dosage d'éléments légers (magnésium, sodium, oxygène, carbone) que n'autorise pas - ou mal - la technique utilisée ici. D'autre part, dans les cas où les informations voulues peuvent être trouvées, une étude historique aussi bien sur les objets que sur l'aspect technique précis des procédés pourrait permettre d'affiner l'attribution des différentes compositions, et de comprendre leur origine.

\section{Remerciements}

Les auteurs remercient chaleureusement les différents Musées sollicités, qui ont tous accepté de participer à cette étude en mettant à leur disposition une ou plusieurs pièces en aluminium de leur collection : (par ordre alphabétique) les Arts décoratifs, le Musée de l'Air et de l'Espace, le Musée de l'Armée, le Musée des Arts et Métiers, les Musée National du Château de Compiègne, et le Musée d'Orsay.

Les auteurs remercient également le Centre de Recherches ALCAN à Voreppe, qui a bien voulu leur céder sept matériaux de référence certifiés pour valider les analyses.

Un grand merci à l'équipe d'AGLAE pour son assistance lors des mesures, et à Benoît Mille pour le suivi accordé à cette étude depuis le début jusqu'à la relecture du manuscrit.

\section{Bibliographie}

CAlligaro, T., DRAN, J.C., haMON, H., MOIGNARD, B. et SALOMON, J., 1998 - An external milli-beam for archacometric applications on the AGLAE IBA facility of the Louvre museum, Nuclear Instruments and Methods, B 136-138: 339-343.

DRAN, J.-C., CALligaRo, T. et SALOMON, J., 2000 - Particleinduced X-ray emission, in E. Ciliberto, G. Spoto (eds.) Modern Analytical Methods in Art and Archaeology, John Wiley, Chichester, 135-166.

FIGUIER, L., 1867-1869 - Les merveilles de la science, Furne, Jouvet ct co, Paris

HAMMER, P., 2005 - Communication privée.

LEJEAL, A., 1894 - L'aluminium. J.-B. Baillère et Fils, Paris.

LEROY, M., 2005 - Communication privéc.

MAZARD, J., 1969 - Histoire monétaire et numismatique contemporaine. Tome 2 . Bourgey, Paris : 166.

Pl.ATEAU, J., 2003 - La naissance de l'aluminium, Techné, 18 : 37-42.

RÉGNIER, J., 1989 - Naissance de l'cxploitation de la bauxite en France. Cahiers d'histoire de l'aluminium, $5: 9-15$.

SAINTE-CLAIRE DEVILLE, H., 1859 - De l'aluminium. MalletBachelicr, Paris.

SCHMITT, L., 2005 - Communication privée (Comptoir général de bourse CGB) 